find that the transition in overall stiffness, $G$, from stretching-dominated behaviour, with $G \propto \mu$, to the bending-dominated regime, where $G \propto \kappa / l^{2}$, occurs in a coupled regime for which $G \propto \mu^{1-x}\left(\kappa / l^{2}\right)^{x}$, with an exponent $x$ that is related to the network topology.

The generality of this result speaks to its importance for a wide range of fibre networks connected by rotating joints. Biophysical networks (like the cytoskeleton, for example) fall naturally into this category - comprising long, semi-flexible filaments connected by crosslinking proteins. These crosslinkers differ depending on the network, but in general they tend to be quite large.

Their size questions the likelihood that a crosslink can rotate freely while remaining stiff with respect to filament displacement, as is assumed in the model proposed by Broedersz et al. ${ }^{1}$. Perhaps a more realistic interpretation of bending stiffness in biophysical networks should include that of both filament and crosslink. Of course, this implies that the bending stiffness depends sensitively on network structure, which complicates application of the theory. An extension that distinguishes the bending stiffness of filaments from that of their crosslinkers is likely to be the subject of future studies, for which the present results offer a key source of inspiration and comparison.

Erik van der Giessen is in the Department of Applied Physics at the University of Groningen, 9747 AG Groningen, The Netherlands. e-mail:E.van.der.Giessen@rug.nl

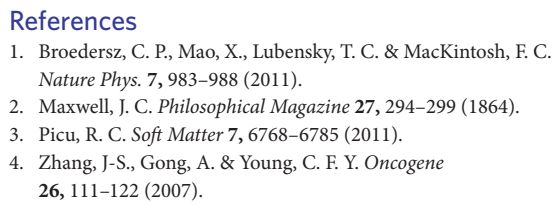

Published online: 30 October 2011

\title{
SELF-SIMILARITY
}

\section{The answer, my friend}

If you've ever spent time gazing up through a tree's foliage, you may well have noticed that branch thickness seems to obey a kind of conservation principle from root to leaf. History marks Leonardo da Vinci as having been the first to put this observation into writing. His notebooks record his reflection that the thicknesses of a tree's branches sum to that of its trunk, at every stage of its growth. More precisely, he claimed that the squared diameter of the trunk equates to the sum of the squared diameters of the ascendant branches.

Although the equality remains unverified, and the exact exponent the subject of some discussion, da Vinci's rule is widely regarded in the world of computer graphics as an effective means of generating realistic trees. Now, Christophe Eloy has gone beyond intuition and aesthetics to probe the physics underpinning the

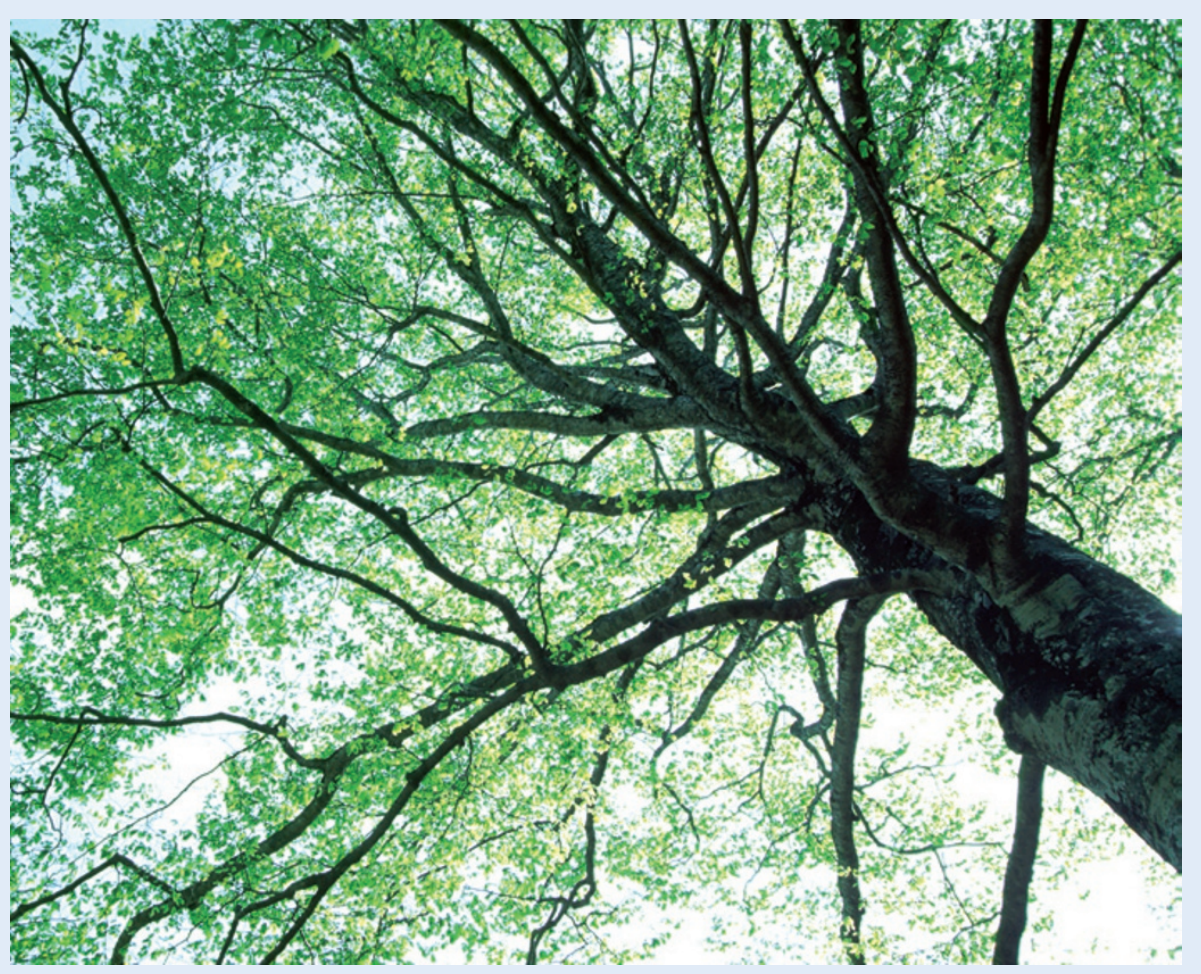

observation - suggesting that the rule follows naturally from a mechanism that helps the tree withstand windinduced stresses (http://arxiv.org/ abs/1105.2591; 2011).

The relationship between a tree's response to wind and the diameter of its trunk was noticed back in the nineteenth century, when Metzger proposed that trunk thickness might be tuned to maintain constant bending stress. Eloy has applied a similar idea to the task of understanding da Vinci's observation. Drawing on the underlying self-similarity of the tree skeleton, his study brings a continuous model for wind loading together with a discrete picture relating a tree's dimensions at each rank to its fractal dimension and the unknown exponent in da Vinci's equation. By assuming that wind acts on the tree's foliage, and that the probability of stress-induced fracture is uniform, Eloy has recovered da Vinci's elegant rule.

A three-dimensional numerical model that factors in the asymmetry and stochasticity of branching, and the variability in the angle of incoming wind, attests to the robustness of Eloy's theory, which accurately predicts the numerically calculated branch diameters and the exponent in da Vinci's relation. The results seem to suggest that da Vinci's insightful observation corresponds to an optimal geometry for resisting wind-induced fracture in selfsimilar trees. 